\title{
Low Impulsive Action, but not Impulsive Choice, Predicts Greater Conditioned Reinforcer Salience and Augmented Nucleus Accumbens Dopamine Release
}

\author{
Fiona D Zeeb ${ }^{*, 1,2}$, Ashlie D Soko', Xiaodong Ji' and Paul J Fletcher ${ }^{1,2,3}$ \\ 'Section of Biopsychology, Campbell Family Mental Health Research Institute, Centre for Addiction and Mental Health (CAMH), Toronto, \\ ON, Canada; '2Department of Psychiatry, University of Toronto, Toronto, ON, Canada; ${ }^{3}$ Department of Psychology, University of Toronto, Toronto, \\ ON, Canada
}

\begin{abstract}
Poor impulse control is associated with an increased propensity to develop an addiction and may contribute to relapse as high impulsive subjects appear to attribute greater salience toward drug-paired stimuli. In these studies, we determined whether trait impulsivity also predicts the desire to obtain natural reward-paired stimuli. Rats trained on the 5-choice serial reaction time task to measure impulsive action (Experiment I) or a delay-discounting task to measure impulsive choice (Experiment 2) were separated into low, intermediate, or high impulsive action ( $L-I A, I-I A, H-I A$ ) or choice (L-IC, I-IC, H-IC) groups. The motivation to obtain a conditioned stimulus (CS) paired with water-reward was subsequently determined by measuring responding for the CS as a conditioned reinforcer (CRf). Dopamine release in the nucleus accumbens was also measured using in vivo microdialysis. The effects of amphetamine were assessed on all tests. In Experiment I, amphetamine increased impulsive action in all groups. L-IA rats initially demonstrated the highest responding for the CRf. Amphetamine increased responding for the CRf and this effect was augmented in L-IA rats. Dopamine release following amphetamine was greatest in L-IA subjects. In Experiment 2, amphetamine increased impulsive choice for L-IC and I-IC rats. However, all groups responded similarly for the CRf and dopamine release was moderately greater in L-IC rats. In conclusion, impulsive choice was unrelated to responding for a CRf. L-IA subjects initially attributed enhanced salience to a CS and exhibited greater dopamine release. Lower dopamine release in $\mathrm{H}-\mathrm{IA}$ rats could result in reduced reinforcing properties of the CRf.

Neuropsychopharmacology (2016) 4I, 209I-2100; doi:I0.1038/npp.2016.9; published online I0 February 2016
\end{abstract}

\section{INTRODUCTION}

Abnormally high levels of impulsivity-broadly defined as action without foresight-are associated with an increased propensity to develop an addiction and greater likelihood of relapse (Jupp and Dalley, 2014; Stevens et al, 2014). Two main categories of impulsivity are impulsive action (an inability to refrain from making a prepotent motor response) and impulsive choice (an inability to wait for reward). Although they can be neuroanatomically and neurochemically dissociated (Pattij and Vanderschuren, 2008), both forms of impulsivity are linked with addiction. For example, high impulsive choice (Perry et al, 2005) or high impulsive action (Dalley et al, 2007, but also see Economidou et al, 2009) was associated with greater cocaine self-administration. However, only high impulsive action predicted enhanced self-administration of nicotine (Diergaarde et al, 2008) but

\footnotetext{
*Correspondence: Dr FD Zeeb, Section of Biopsychology, Centre for Addiction and Mental Health (CAMH), Campbell Family Mental Health Research Institute, 250 College St, Toronto, M5T IR8 ON, Canada, Tel: + I 416535 850।, E-mail: fiona.zeeb@camh.ca

Received 5 November 2015; revised II January 2016; accepted 12 January 20 I6; accepted article preview online 19 January 2016
}

not heroin self-administration (McNamara et al, 2010). Therefore, although there is clear evidence of an impulsivityaddiction relationship, it may be influenced by the specific drug of abuse and type of impulsivity.

Stimuli associated with drug taking strongly influence drug-taking and drug-seeking behavior. Specifically, cigarette smoking is largely reinforced by cues directly associated with cigarette use (Caggiula et al, 2001; Rose et al, 2000). Furthermore, exposure to drug-paired stimuli elicited craving and automatic drug-seeking responses in abstinent individuals (Tiffany and Carter, 1998; Volkow et al, 2006). In preclinical research, addiction-related behavior can be studied using the self-administration procedure, during which animals make operant responses to receive drug infusions often paired with a stimulus (eg, light and/or tone). The influence of drug-paired conditioned stimuli (CSs) on behavior is then measured when animals respond only for the stimuli during extinction or subsequent reinstatement tests.

High impulsive choice-but not action-predicted greater responding following re-exposure to nicotine-paired cues during a reinstatement test (Diergaarde et al, 2008). In contrast, rats with high levels of impulsive action responded 
more for a cocaine-paired stimulus following punishmentinduced abstinence (Economidou et al, 2009). Likewise, high impulsive action was related to greater seeking responses directed at a sucrose-paired stimulus (Diergaarde et al, 2009). Notably, these preclinical studies demonstrated that poor impulse control is not necessarily an artifact of chronic substance abuse. In sum, although the relationship may be dependent on the specific drug of abuse, both impulsive action and choice are associated with the propensity to relapse due to the salience attributed to drug-paired stimuli.

The notion that impulsivity may influence the attribution of salience to stimuli (thereby promoting addiction maintenance and relapse), is derived from experiments that specifically focus on stimuli directly paired with selfadministered food or drug reward. It is not clear whether trait impulsivity predicts the attribution of salience toward all reward-paired stimuli. The present study determined whether different types of trait impulsivity predict the motivation to seek stimuli paired with a natural, not selfadministered reward. Findings from these experiments would demonstrate whether highly impulsive subjects exhibit a generalized impairment in over-attributing salience to stimuli.

In separate studies, animals were screened for trait impulsive action (Experiment 1) or choice (Experiment 2). Impulsive action was assessed by measuring premature responses on the 5-choice serial reaction time task (5CSRTT; Robbins, 2002); impulsive choice was indexed by a preference for a smaller, immediate reward over a large, delayed reward using a delay-discounting task (DDT) (Evenden and Ryan, 1996). All rats were then exposed to repeated pairings of a water reward and a light-tone cue (ie, a CS). The salience attributed to the CS was determined by measuring responding for the CS as a conditioned reinforcer (CRf).

Compared with low impulsive subjects, dopamine (DA) D2 receptor density in the nucleus accumbens (NAc) was decreased in high impulsive action rats (Dalley et al, 2007). Likewise, human subjects with high trait impulsivity, compared with control subjects, demonstrated decreased midbrain DA D2/D3 receptor binding (Buckholtz et al, 2010) and greater delay-discounting in pathological gamblers was also associated with decreased striatal DA D2/D3 receptor binding (Joutsa et al, 2015). Furthermore, reduced electrically evoked DA in the NAc was observed in vitro in high impulsive choice and high impulsive action rats (Diergaarde et al, 2008). However, it is unknown if trait impulsivity-action or choice-influences in vivo DA release.

Amphetamine is a monoamine releaser and transporter inhibitor, with a strong affinity for the DA transporter. DA also has an important role in both impulsive action and impulsive choice (Pattij and Vanderschuren, 2008; Robbins, 2002). Additionally, direct infusion of amphetamine in the NAc increased responding for a CRf in control animals, but not in animals with depleted NAc DA (Olmstead et al, 1998; Taylor and Robbins, 1986). We therefore examined the effects of amphetamine on both tests of impulsivity, responding for a CRf at baseline, during extinction, and reinstatement, and used in vivo microdialysis to measure DA release in the NAc.

\section{MATERIALS AND METHODS}

\section{Subjects}

Subjects were male Long Evans rats (Charles River Laboratories, St Constant, QC, Canada), weighing 275$300 \mathrm{~g}$ at the beginning of the study, pair-housed until surgery. Experiment 1 used 36 rats; Experiment 2 used 30 rats. Animals were food-restricted (85-90\% of free-feeding weight) during impulsivity testing; otherwise food was freely available. Water was available ad libitum except during conditioned reinforcement testing, in which access was restricted to $2 \mathrm{~h} /$ day. Behavioral testing occurred 4-6 times per week. Colony rooms were maintained at $21^{\circ} \mathrm{C}$ under a $12 \mathrm{~h}$ light cycle (lights on at 0800 hours). Experiments were conducted in accordance with the Canadian Council of Animal Care, and the Centre for Addiction and Mental Health Animal Care Committee approved all protocols.

\section{Impulsivity Testing}

Descriptions of the operant conditioning chambers and protocol details are provided in the Supplementary Materials and Methods.

5CSRTT (Experiment 1). Briefly, rats initiated a trial by making a nosepoke response into the food tray. After a $5 \mathrm{~s}$ intertrial interval (ITI), one of five response holes along the opposite wall of the chamber was briefly illuminated. A response in this hole turned off the stimulus light, turned on the traylight, and delivered a pellet reward ( $45 \mathrm{mg}$, Bioserv, Flemington, New Jersey). A nosepoke into a response hole during the ITI was classified as a premature response (indicative of impulsive action; Robbins, 2002).

Once performance was stable, rats were separated into high (H-IA, $n=9)$, intermediate (I-IA, $n=18)$, and low (L-IA, $n=9$ ) impulsive action groups based on quartile analysis of the average percentage of premature responses across the last three baseline sessions. Quartile analyses was used as the number of premature responses made at baseline followed a continuum. Animals were then tested in two separate sessions with an increased ITI duration (7 $\mathrm{s}$ and $9 \mathrm{~s}$ ). Rats then returned to the standard $5 \mathrm{~s}$ ITI program and received an injection of amphetamine $(0,0.15,0.3$, or $0.6 \mathrm{mg} /$ $\mathrm{kg}$ ) before testing.

DDT (Experiment 2). Each 56-min session consisted of 4 blocks of 12 trials (Zeeb et al, 2010). The first 2 trials were forced choice, in which only one lever was extended. Both levers were extended for the following 10 trials. A trial began when the houselight turned on and lever(s) extended $2 \mathrm{~s}$ later. A response on one lever immediately delivered a small reward ( 1 pellet; $45 \mathrm{mg}$, Bioserv). A response on the opposite lever initiated a delay, after which 4 pellets were delivered. The delay to the large-reward began at $0 \mathrm{~s}$ and increased in $15 \mathrm{~s}$ intervals after each block of trials. Trials lasted for $70 \mathrm{~s}$ regardless of choice.

When choice preference was stable, animals were separated into high (H-IC, $n=13$ ), intermediate (I-IC, $n=8$ ), and low (L-IC, $n=9$ ) impulsive choice groups based on the average preference for the large reward across the last three baseline sessions once the delay was introduced (ie, choice 
during block 2; Zeeb et al, 2010). There was a clear distinction in choice of the large reward in block 2 between animals, therefore the following criteria was used: L-IC rats chose the large reward $\geqslant 7$ times, $\mathrm{H}$-IC rats chose the large reward $\leqslant 3$ times, and the remaining animals were designated I-IC rats. Animals then received an injection of amphetamine $(0,0.15,0.3$, or $0.6 \mathrm{mg} / \mathrm{kg})$ before testing. As these doses did not affect impulsive choice (Supplementary Figure S2), rats were subsequently challenged with two higher doses $(0,1.0,1.5 \mathrm{mg} / \mathrm{kg})$.

\section{Responding for a CRf}

One week after impulsivity testing finished, rats were trained to respond for a CRf in operant conditioning chambers different from those used for impulsivity testing. Additional details are described in Supplementary Materials and Methods. Rats first received 10 daily Pavlovian conditioning sessions. In each session, animals were exposed to 30 presentations of a 5-s CS immediately followed by delivery of $0.05 \mathrm{ml}$ water reward. During the next phase, two levers (Experiment 1) or nosepoke ports (Experiment 2) were present. Responding on one lever/nosepoke port delivered the CS-now considered a CRf-according to a random ratio 2 schedule. Responses on the opposite lever/nosepoke port (NCRf) had no consequences. Levers/nosepoke ports were available throughout the 40 -min session.

Responding for the CRf was measured across five baseline sessions. Rats then received an injection of amphetamine ( 0 , $0.15,0.3$, or $0.6 \mathrm{mg} / \mathrm{kg}$ ) before testing. Subsequently, animals were tested for three sessions (in which response rates did not significantly differ from the pre-amphetamine baseline session) before receiving six extinction sessions during which responding on either lever/nosepoke port had no consequence. The effect of amphetamine $(0,0.3 \mathrm{mg} / \mathrm{kg})$ was tested over 2 additional extinction sessions followed by 2 'reinstatement' sessions, in which responding on the CRf lever/ nosepoke port again delivered the CRf.

\section{In Vivo Microdialysis}

See Supplementary Materials and Methods for additional details. Following conditioned reinforcement testing, H-IA, H-IC, L-IA, and L-IC rats were surgically implanted with a cannula aimed at the left NAc. After a recovery period (minimum of 5 days), probes (MAB 9.14.2; $2 \mathrm{~mm}$ membrane; Scientific Products, Toronto, ON, Canada) that traversed both the NAc core and shell sub-regions were lowered into the guide. Dialysate samples were collected every $10 \mathrm{~min}$ and analyzed immediately using highperformance liquid chromatography (HPLC) coupled with electrochemical detection. Once a stable baseline was detected, animals received an injection of saline and five samples were collected. Rats then received an injection of $0.3 \mathrm{mg} / \mathrm{kg}$ amphetamine and 12 samples were collected, followed by an injection of $0.6 \mathrm{mg} / \mathrm{kg}$ amphetamine and 15 samples were collected. Sample collection began immediately following an injection. DA concentrations were calculated as a percent change from baseline (Justice, 1993; Feenstra 2007).

Rats were killed using an increasing concentration of $\mathrm{CO}_{2}$ immediately following the microdialysis session. Brains were removed and post-fixed in a $4 \%$ formaldehyde solution, then transferred to a $30 \%$ sucrose solution. $40 \mu \mathrm{m}$ sections throughout the NAc were stained with cresyl violet to verify the probe location (Supplementary Figure S5).

\section{Amphetamine}

d-amphetamine sulfate (U.S. Pharmacopeia, Rockville, MD) was dissolved in sterile saline and administered intraperitoneally. Doses were calculated as a base. During behavioral testing, injections occurred $10 \mathrm{~min}$ before testing and test sessions took place twice a week, a minimum of $48 \mathrm{~h}$ apart. For impulsivity testing, at least one baseline session preceded an amphetamine test session. Doses were administered according to a Latin Square design. If saline and one dose were used, administration was counterbalanced across rats.

\section{Statistical Analysis}

Using SYSTAT for Windows (version 12.00.08), data were analyzed by a repeated-measures ANOVA with the Impulsivity group as a between-subjects factor. Withinsubjects factors included session, delay, dose of amphetamine, response (CS vs Pre-CS), side (CRf vs NCRf), or sample. Post hoc analysis was conducted using paired-sample $t$-tests to compare data within a group and two-sample $t$-tests to compare data between groups. A $P$-value of $\leqslant 0.05$ denoted significance. If a dose $\times$ side interaction was observed when animals were responding for a CRf, planned comparisons using paired-sample $t$-tests were performed within each group as we hypothesized that amphetamine would increase responding only on the active lever similar to previous research (eg, Browne et al, 2014; Robbins et al, 1983; Winterbauer and Balleine, 2007). An arcsine transformation was performed prior to statistical analysis of variables from behavioral testing expressed as a percentage to limit the effect of an artificially imposed ceiling (McDonald, 2014).

\section{RESULTS}

\section{Experiment 1}

5CSRTT. Premature responding was significantly different between L-IA, I-IA, and H-IA rats (Figure 1a; impulsivity: $\left.\mathrm{F}_{2,33}=60.061, P<0.001\right)$. These differences emerged during training and were stable (Supplementary Figure S1). Similar to previous research (Economidou et al, 2009), L-IA rats were more accurate on the 5CSRTT compared with I-IA and H-IA groups, however, the main effect of impulsivity just failed to reach significance (Supplementary Table S1; impulsivity: $\left.\mathrm{F}_{2,33}=3.101, P=0.06\right)$.

Compared with each group's own baseline, premature responding increased across all groups when the ITI increased to $7 \mathrm{~s}$ (Figure 1b; session: $\mathrm{F}_{1,33}=12.537$, $P=0.001$; session $\times$ impulsivity: $F_{2,33}=1.499$, not significant (NS)). When the ITI was $9 \mathrm{~s}$, the increase in premature responding from baseline was smallest in $\mathrm{H}$-IA rats compared with the L-IA and I-IA groups (session $\times$ impulsivity: $\mathrm{F}_{2,33}=3.763, P=0.03$; L-IA vs $\mathrm{H}$-IA: $\mathrm{F}_{1,16}=$ 4.22, $P=0.06$, I-IA vs H-IA: $\mathrm{F}_{1,25}=7.013, P=0.01$ ). Furthermore, with a $9 \mathrm{~s}$ ITI there was no longer a difference between I-IA and $\mathrm{H}$-IA rats $\left(\mathrm{F}_{1,25}=0.660, \mathrm{NS}\right)$, indicating a potential ceiling effect. Amphetamine increased premature responding 

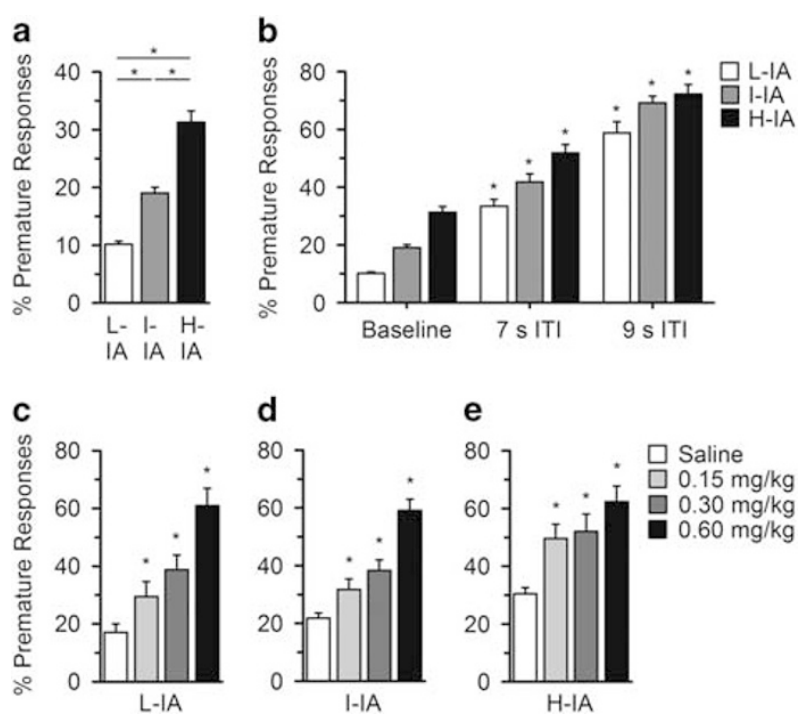

Figure I Baseline levels of impulsive action and influence of lengthening the ITI and amphetamine administration. (a) Average number of premature responses made during the last three baseline sessions. $\mathrm{H}-\mathrm{IA}$ rats made the greatest number of premature responses, followed by I-IA rats and L-IA rats $(H-|A>|-|A>L-| A)$. Lengthening the ITI to $9 s$ or $7 s$ from $5 s$ increased premature responding in the L-IA, I-IA, and H-IA groups. (b) With a $7 \mathrm{~s} I T I$, there were still clear differences between the three groups. With a $9 \mathrm{~s} I T I$, there was no longer a group difference between the I-IA and H-IA groups, however, the L-IA group still made the least number of premature responses $(H-|A=|-|A>L-| A)$. Amphetamine increased premature responding in the L-IA (c), I-IA (d), and H-IA (e) groups. Data represent an average \pm SEM. * indicates significant result from post hoc analyses comparing behavior (a) between groups, (b) between baseline and the ITI challenges, or $(\mathrm{c}-\mathrm{e})$ between saline and amphetamine doses.

in all groups (Figure 1c-e; All groups, all doses: dose: $\mathrm{F}_{3,99}=77.919, \quad P<0.001 ;$ dose $\times$ impulsivity: $\mathrm{F}_{6,99}=1.804$, NS). Results from the other variables measured are in Supplementary Table S1.

Responding for conditioned reinforcement. During Pavlovian conditioning, all groups responded minimally when the CS was absent and increased responding during CS presentation (Figure 2a; response: $\mathrm{F}_{1,33}=225.029, P<0.001$; session $\times$ response: $\quad \mathrm{F}_{9,297}=34.011, \quad P<0.001 ; \quad$ session $\times$ response $\times$ impulsivity: $\left.F_{18,297}=0.615, N S\right)$. The total number of responses made during these sessions and the latency to collect the reward also did not differ between groups (Supplementary Figure S3).

When given the opportunity to respond for the CS (now a CRf), rats responded more on the lever delivering the CRf than the inactive, NCRf lever (side: $\mathrm{F}_{1.33}=46.698, P<0.001$; side $\times$ impulsivity: $F_{2,33}=1.849$, NS). Interestingly, L-IA rats initially responded more for the CRf compared with I-IA and H-IA groups (Figure $2 \mathrm{~b}$; session $\times$ side $\times$ impulsivity: $\left.\mathrm{F}_{8,132}=1.986, P=0.05\right)$. This effect was greatest during the second (impulsivity: L-IA vs H-IA: $\mathrm{F}_{1,16}=5.561, P=0.03$; L-IA $v s$ I-IA: $\mathrm{F}_{1,16}=6.136, P=0.02$ ) and third (impulsivity: L-IA $v s$ H-IA: $\mathrm{F}_{1,16}=3.891, \quad P=0.07$; L-IA $\quad v s$ I-IA: $\left.\mathrm{F}_{1,16}=4.881, P=0.04\right)$ day of testing. There was no difference between the groups on the fourth or fifth day of testing, demonstrating that this was a transient effect.
Amphetamine selectively increased responding on the CRf lever (Figure $2 \mathrm{c}$; dose $\times$ side: $\mathrm{F}_{3,99}=24.548, P<0.001$ ). L-IA rats demonstrated the greatest increase compared with both I-IA and H-IA groups, which exhibited similar response rates (dose $\times$ impulsivity-all groups: $\mathrm{F}_{6,99}=2.368, P=0.04$; L-IA $v s$ I-IA: $\mathrm{F}_{3,75}=2.893, \quad P=0.04 ; \quad$ L-IA $v s$ H-IA: $\mathrm{F}_{3,48}=3.652, P=0.02$; I-IA $v s$ H-IA: $\mathrm{F}_{3,75}=0.444$, NS).

Across 6 days of extinction (no CRf delivery) responding decreased in all groups (Supplementary Figure S4). During extinction, amphetamine increased responding on the lever that previously delivered the CRf (Figure 2d; dose $\times$ side: $\mathrm{F}_{1,32}=23.910, \quad P<0.001 ; \quad$ dose $\times$ side $\times$ impulsivity: $\mathrm{F}_{2,32}=1.484$, NS). However, results from planned comparisons revealed that this increase was NS in H-IA rats (Figure 2d). During reinstatement (in which the CRf was again delivered response-contingently), amphetamine increased responding on the $\mathrm{CRf}$ lever (dose $\times$ side: $\mathrm{F}_{1,32}=6.494, P=0.02$; dose $\times$ side $\times$ impulsivity: $\mathrm{F}_{2,32}=1.251$, $\mathrm{NS})$, and analyses from planned comparisons determined that this effect was only significant in L-IA rats (Figure 2e). Although there was a significant dose $\times$ lever interaction in the L-IA group $\left(\mathrm{F}_{1,8}=14.391, P=0.005\right)$, responding increased on both the CRf and NCRf lever. Together, these results demonstrate that amphetamine increases the motivation to obtain the CRf and L-IA rats are more sensitive to amphetamine's effects.

Microdialysis. Similar to previous reports (Dalley et al, 2007), there was no significant group difference in the concentration of extracellular DA detected at baseline (Supplementary Table S3). Amphetamine increased DA release in the NAc (sample-0.3 mg/kg: $F_{11,110}=5.456$, $\left.P<0.001 ; \quad 0.6 \mathrm{mg} / \mathrm{kg}: \mathrm{F}_{14,140}=8.774, P<0.001\right)$ and this increase was significantly larger for L-IA rats compared with $\mathrm{H}-\mathrm{IA}$ rats (Figure $3 \mathrm{a}$; sample $\times$ impulsivity $-0.3 \mathrm{mg} / \mathrm{kg}$ : $\mathrm{F}_{11,110}=1.948, P=0.04 ; 0.6 \mathrm{mg} / \mathrm{kg}: \mathrm{F}_{14,140}=1.846, P=0.04$ ).

\section{Experiment 2}

DDT. All rats discounted the large-reward option as the delay to receiving this reward increased, and H-IC rats demonstrated the steepest discounting rate (Figure 4a; delay: $\mathrm{F}_{3,81}=179.843, P<0.001$; delay $\times$ impulsivity: $\mathrm{F}_{6,81}=20.396$, $P<0.001)$. Amphetamine increased impulsive choice in L-IC and I-IC rats, but not $\mathrm{H}-\mathrm{IC}$ rats (Figure $4 \mathrm{~b}-\mathrm{d}$; dose $\times$ impulsivity: $\quad \mathrm{F}_{4,54}=5.577, \quad P=0.001 ;$ dose $\times$ delay-L-IC: $\mathrm{F}_{6,48}=3.753, P=0.004 ; \mathrm{I}-\mathrm{IC}: \mathrm{F}_{6,42}=2.359, P=0.05 ; \mathrm{H}-\mathrm{IC}$ : $\left.\mathrm{F}_{6,72}=2.138, \mathrm{NS}\right)$. The lack of effect in the H-IC group may be partially caused by a ceiling effect, as these rats were already very impulsive. Results from the other variables measured during the DDT are in Supplementary Table S2.

Conditioned reinforcement. Responses during the CS presentation increased across Pavlovian training and animals made few responses when the CS was absent (Figure 5a; sessions 1-10, CS vs Pre-CS-response: $\mathrm{F}_{1,27}=125.588$, $P<0.001$; response $\times$ impulsivity: $\mathrm{F}_{2,27}=0.453$, NS). The total number of responses made and the latency to collect the reward also did not differ between groups (Supplementary Figure S3). 

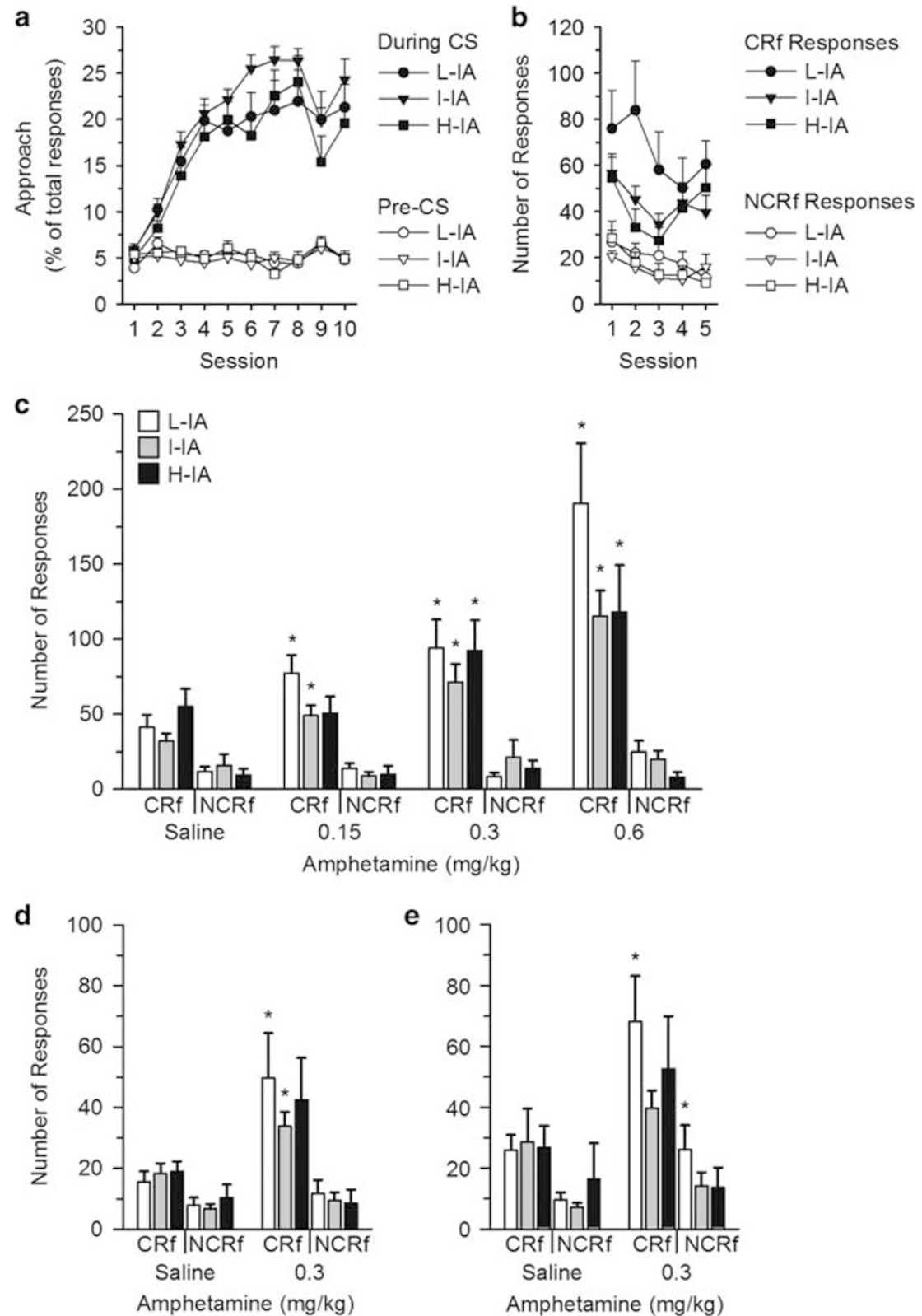

Figure 2 Impulsive action influences responding for a CRf. (a) During Pavlovian conditioning, animals in all groups made a similar number of responses during the Pre-CS period, and the number of approach responses in the reward receptacle increased when the CS was present across the 10 Pavlovian conditioning sessions. (b) Animals in the L-IA group initially responded more on the lever delivering the CRf compared with both $\mathrm{H}$-IA and I-IA groups. All rats demonstrated a preference for the CRf lever over the inactive (NCRf) lever. (c) Compared with saline, amphetamine selectively increased responding on the CRf lever in all groups; however, this effect was augmented in the L-IA group. (d) During an extinction test, amphetamine increased responding on the lever that previously delivered the CRf. This increase was only significant in the L-IA and I-IA groups. (e) Amphetamine again increased responding during a 'reinstatement' test (responding on the CRf lever delivered the CRf). Planned comparisons demonstrated that responding significantly increased only in the L-IA group on both the CRf and NCRf levers; however, there was still a preference for the CRf lever over the NCRf lever. Data represent an average \pm SEM. * indicates significant result from the paired-sample $t$-tests comparing saline and amphetamine within each group.

During tests of responding for the CRf, all rats preferentially responded in the CRf nosepoke port than the NCRf nosepoke port (Figure 5b; side: $F_{1,27}=53.603, P<0.001$; side $\times$ impulsivity: $\mathrm{F}_{2,27}=0.901$, NS; session $\times$ side $\times$ impulsivity: $\mathrm{F}_{8,108}=0.948$, NS). Amphetamine increased responding selectively in the CRf nosepoke port in all groups (Figure 5c: dose $\times$ side: $F_{3,81}=13.728, \quad P<0.001$; dose $\times$ impulsivity: $\mathrm{F}_{6,81}=0.529, \mathrm{NS}$ ).
Animals comparably reduced responding during extinction (Supplementary Figure S4). Amphetamine increased responding in the inactive $\mathrm{CRf}$ nosepoke port during extinction (Figure 5d; dose $\times$ side: $\mathrm{F}_{1,27}=25.043, P<0.001$; dose $\times$ side $\times$ impulsivity: $F_{2,27}=1.019, \mathrm{NS}$ ) and in the again active CRf nosepoke port during reinstatement (Figure 5e; dose $\times$ side: $\quad F_{1,27}=33.178, \quad P<0.001 ;$ dose $\times$ side $\times$ impulsivity: $\left.F_{2,27}=1.769, \mathrm{NS}\right)$. During the reinstatement test, 

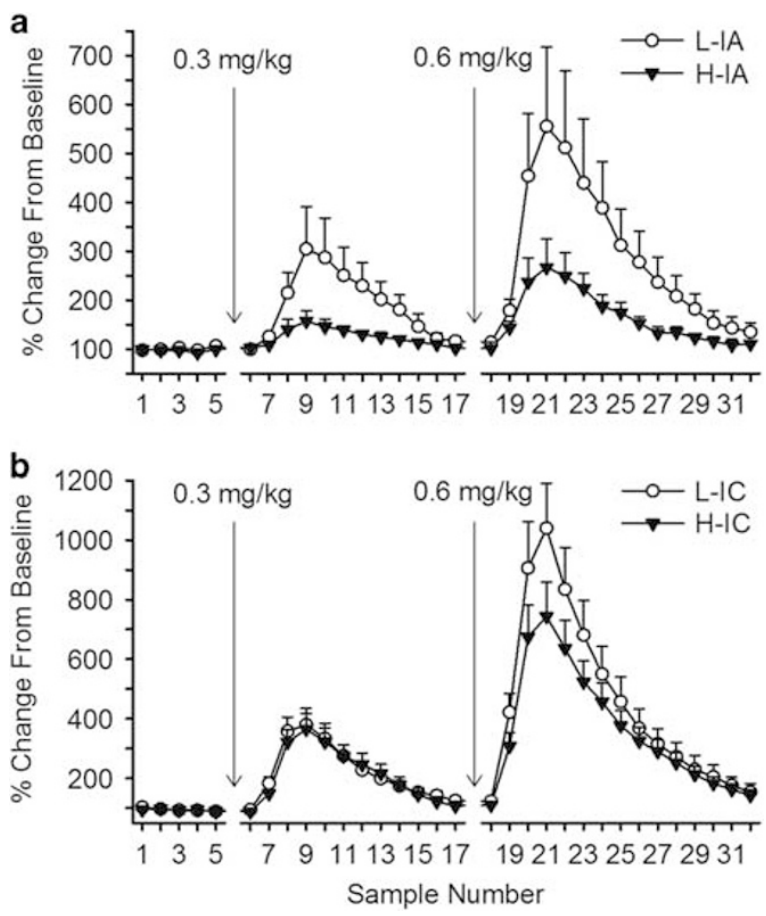

Figure 3 The ability of amphetamine to increase extracellular DA levels is augmented in low impulsive rats. (a) An injection of saline did not alter DA release. Following an injection of either $0.3 \mathrm{mg} / \mathrm{kg}$ or $0.6 \mathrm{mg} / \mathrm{kg}$ amphetamine, extracellular DA significantly increased from baseline levels. The amount of DA measured in L-IA animals was significantly greater than $\mathrm{H}-\mathrm{IA}$ rats. (b) Saline did not alter DA release. A comparable increase in DA concentration was observed between $\mathrm{L}-\mathrm{IC}$ and $\mathrm{H}-\mathrm{IC}$ groups following injection of $0.3 \mathrm{mg} / \mathrm{kg}$ amphetamine. L-IC animals displayed moderately greater release of DA following injection of $0.6 \mathrm{mg} / \mathrm{kg}$ amphetamine. Data are presented as a percentage of baseline levels of DA detected (L-IA: $4.4 \mathrm{nM} \pm \mathrm{I} .5 ; \mathrm{H}-\mathrm{IA}: 7.0 \mathrm{nM} \pm 4.4 ; \mathrm{L}-\mathrm{IC}: 4.8 \mathrm{nM} \pm 3.2 ; \mathrm{H}-\mathrm{IC}: 3.5 \mathrm{nM} \pm 2.0$ ). There was no significant difference in the amount of DA detected at baseline between groups in either experiment (Supplementary Table S3). Data represent an average \pm SEM.

although there was a significant interaction between dose and side for both I-IC $\left(\mathrm{F}_{1,7}=27.179, P<0.001\right)$ and $\mathrm{H}$-IC groups $\left(\mathrm{F}_{1,12}=23.996, P<0.001\right)$, additional analyses revealed a significant increase in both the CRf and NCRf nosepoke ports following amphetamine. Increased responding observed in L-IC rats just failed to reach significance (dose $\times$ side: $\mathrm{F}_{1,8}=4.471, P=0.07$ ).

Microdialysis. Amphetamine increased DA release in the NAc (Figure 3b; sample-0.3 mg/kg: $\quad F_{11,198}=41.427$, $\left.P<0.001 ; 0.6 \mathrm{mg} / \mathrm{kg}: \mathrm{F}_{14,210}=56.255, P<0.001\right)$. Although there was no group difference in extracellular DA concentration following $0.3 \mathrm{mg} / \mathrm{kg}$ amphetamine, L-IC rats demonstrated moderately greater DA release in response to $0.6 \mathrm{mg} / \mathrm{kg}$ amphetamine (sample $\times$ impulsivity $-0.3 \mathrm{mg} / \mathrm{kg}$ : $\mathrm{F}_{11,198}=0.346$, NS; $\left.0.6 \mathrm{mg} / \mathrm{kg}: \mathrm{F}_{14,210}=1.933, P=0.03\right)$.

\section{DISCUSSION}

Here, we demonstrated that reduced trait impulsive action, but not impulsive choice, shares a relationship with the ability of stimuli to support operant responding, likely
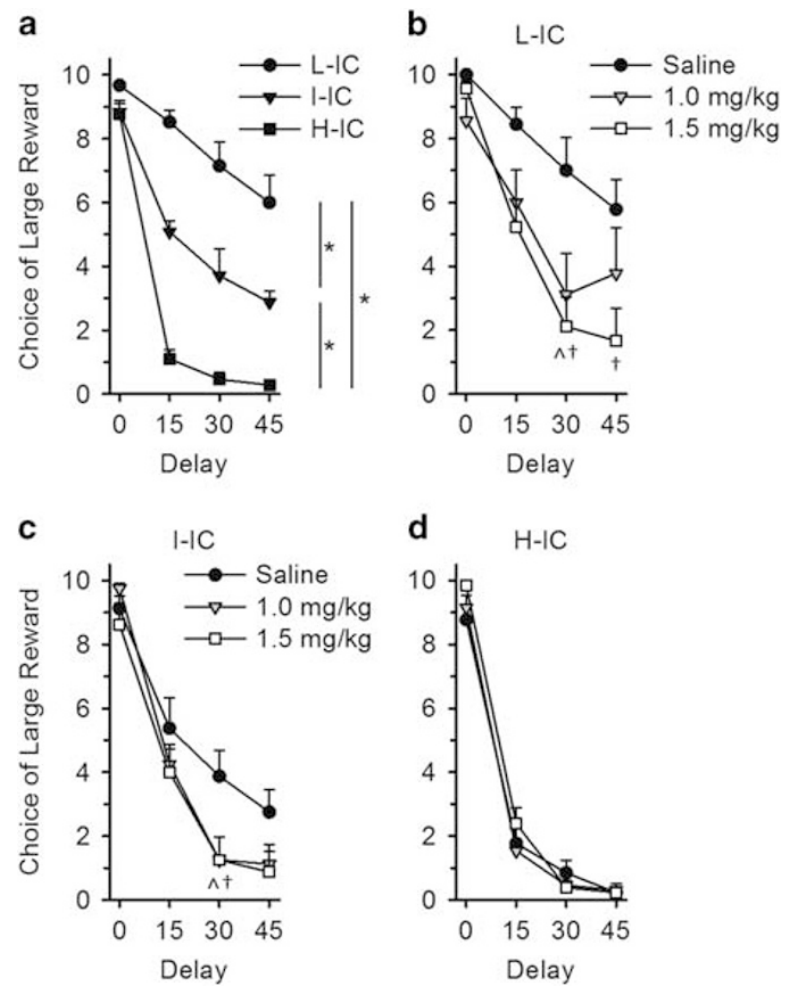

Figure 4 Trait levels of impulsive choice and effects of amphetamine on delay-discounting. (a) Animals in the L-IC group chose the large-reward option significantly more than the $\mathrm{I}-\mathrm{IC}$ and $\mathrm{H}-\mathrm{IC}$ when the delay to the large reward was set at 15, 30, or $45 \mathrm{~s}$. Amphetamine increased impulsive choice (ie, decreased choice of the large-reward option) in the L-IC (b) and I-IC (c) groups, but not the $\mathrm{H}-\mathrm{IC}$ (d) group. Data represent an average \pm SEM. * indicates a significant impulsivity $\times$ delay interaction comparing choice of the large-reward option between groups. Caret indicates a significant result from post hoc analyses comparing saline to the $1.0 \mathrm{mg} / \mathrm{kg}$ amphetamine dose. $†$ indicates a significant result from post hoc analyses comparing saline with the $1.5 \mathrm{mg} / \mathrm{kg}$ amphetamine dose.

through the acquisition of incentive/motivational salience. Trait impulsive choice did not predict responding for a CRf. However, subjects with low levels of impulsive action initially responded more for a CRf than either I-IA or H-IA action rats. The ability of amphetamine to enhance responding for the CRf was also greater for L-IA rats, especially following the $0.6 \mathrm{mg} / \mathrm{kg}$ dose. Moreover, L-IA rats were more responsive to amphetamine during extinction (when responses were not reinforced) and in a reinstatement test, when responding once again delivered the CRf. Consistent with these behavioral findings, L-IA rats demonstrated greater amphetamine-stimulated DA release in the NAc compared with H-IA rats.

The reward deficiency syndrome hypothesis of addiction suggests that, compared with healthy controls, addicted individuals experience less pleasure from reward which drives them to seek excessive amounts of reward (such as drugs of abuse, food, sex, gambling, and so on; Comings and Blum, 2000; Downs et al, 2013). This hypothesis largely centers on reduced DA signaling following receipt of reward, generally attributed to decreased DA D2 receptors in the accumbens (Comings and Blum, 2000). Interestingly, rats demonstrating high levels of impulsive action also have a 

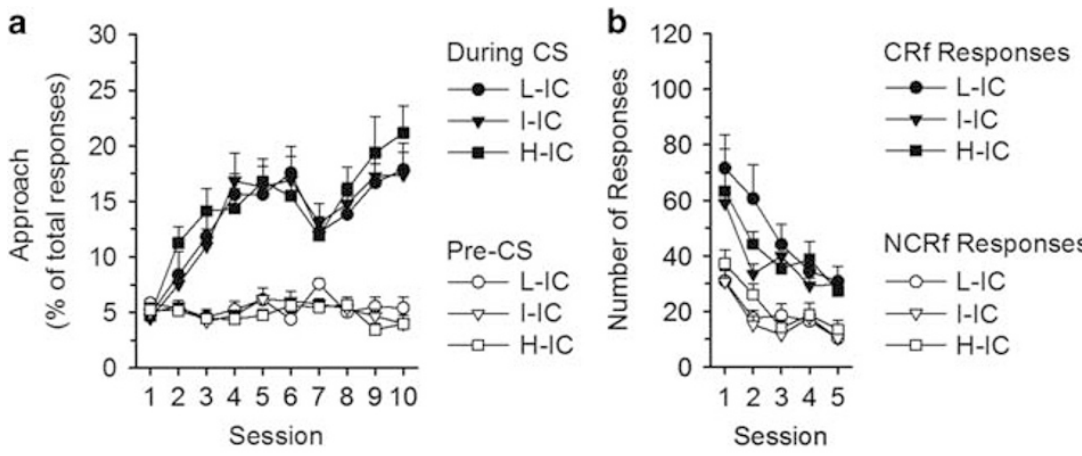

NCRf Responses
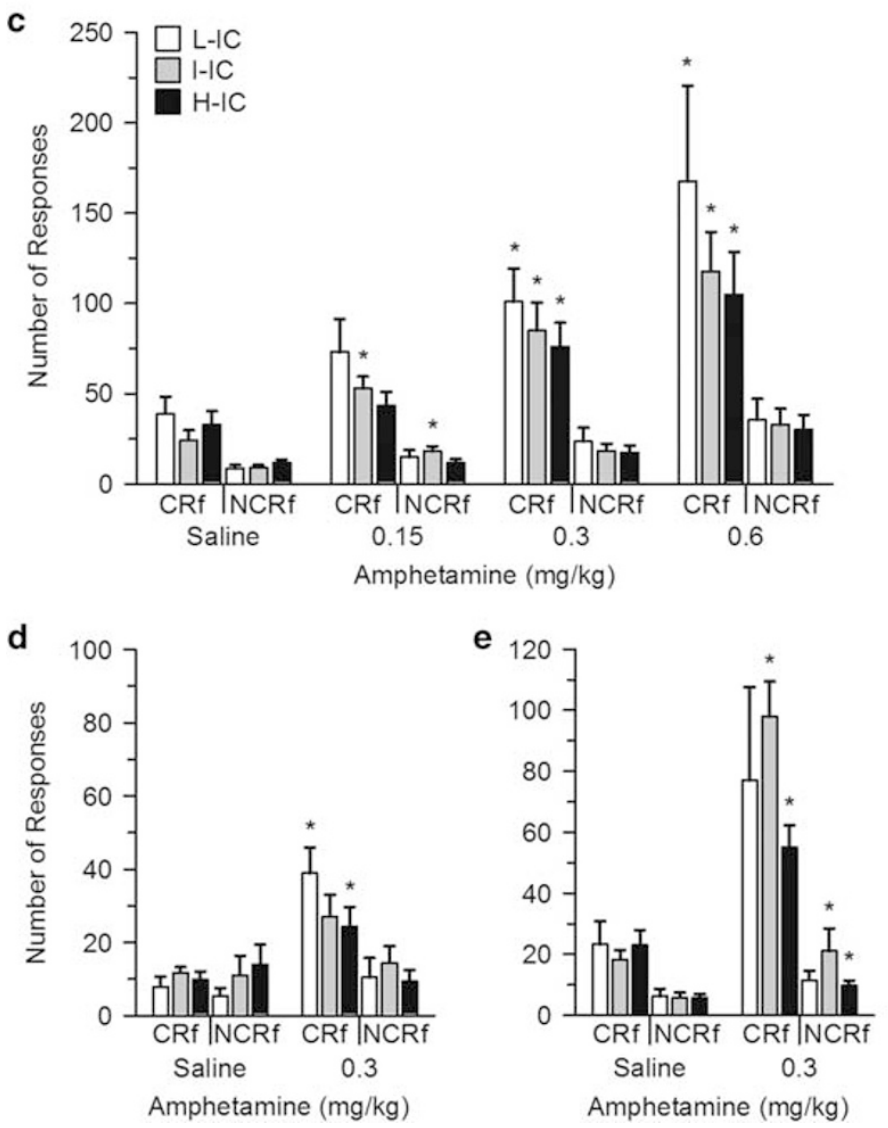

Figure 5 Impulsive choice does not influence responding during Pavlovian conditioning or responding for a CRf. (a) Animals in all groups made a similar number of approach responses when the CS was presented and responded minimally during the Pre-CS period. Responses during CS presentation increased across the 10 sessions of Pavlovian conditioning. (b) Although responding for the CRf decreased across the five baseline test sessions, all groups made a similar number of responses for the CRf and demonstrated a significant preference for the CRf nosepoke port over the NCRf nosepoke port. (c) Amphetamine generally increased responding in the CRf nosepoke port at all doses. (d) During an extinction test, amphetamine again increased responding in the CRf nosepoke port in all groups; however, planned comparisons demonstrated this effect was only significant in the L-IC and H-IC groups. (e) Amphetamine also increased responding during the 'reinstatement' test. Data represent an average \pm SEM. * indicates significant result from the paired-sample $t$-tests comparing saline and amphetamine within each group.

decreased number of DA D2 receptors in the NAc (Dalley et al, 2007). Additionally, in vitro studies have shown that, compared with low impulsive animals, reduced electrically evoked DA in the NAc core is observed in high impulsive choice and high impulsive action rats, whereas reduced electrically evoked DA in the NAc shell was only observed in high impulsive choice rats (Diergaarde et al, 2008). Here, we provide direct evidence for reduced DA release in the NAc following administration of a rewarding drug (amphetamine) in H-IA and H-IC rats.
Similar to previous findings (Dalley et al, 2007), DA levels at baseline did not differ between L-IA and H-IA groups. Therefore, underlying differences in trait impulsivity may not be related to basal extracellular levels of DA, but to mechanisms involved in DA release. If $\mathrm{H}$-IA rats were in a reward-deficient state, we would predict that trait impulsivity would also be positively correlated with increased selfadministration of rewards. This is certainly the case in some situations (Dalley et al, 2007; Diergaarde et al, 2008, 2009), but not others (Economidou et al, 2009; McNamara 
et al, 2010), in addition to responding for the CRf in the present study.

Drug or food self-administration studies have shown that rats with high levels of impulsive action make more seeking responses, demonstrated by heightened responding for reward-paired stimuli in absence of food or drug reward (Diergaarde et al, 2009; Economidou et al, 2009). In the present study, all animals responded for the water-paired stimulus as a CRf, demonstrating that the CS acquired motivational salience and was reinforcing in the absence of primary reward. However, high impulsive subjects did not abnormally seek this secondary reinforcer; H-IA rats did not respond more for the CRf compared with L-IA or I-IA groups. Furthermore, the ability of amphetamine to enhance responding for the CRf was augmented in L-IA subjects, but not in H-IA rats.

It may appear counterintuitive that, compared with low impulsive subjects, high impulsive rats demonstrated increased seeking responses for reward-paired stimuli in selfadministration studies, yet did not respond more for the CRf here. However, this discrepancy could be accounted for by methodological differences. In the present study, animals were passively exposed to an identical number of stimulusreward pairings during Pavlovian conditioning; rats were then required to make a novel instrumental response to receive the CS as a CRf. In contrast, animals tested in a selfadministration paradigm actively respond for reward paired with a stimulus and make the same instrumental response during reinstatement tests. Therefore, seeking responses made during the reinstatement tests are influenced by the animals' previous association of this action and obtaining reward. Although stimuli paired with self-administered drug reward can support a new instrumental response and thus act as a CRf (Di Ciano and Everitt, 2004), whether trait impulsivity influences such responding for a CRf following drug self-administration is unknown. These important distinctions suggest that fundamental differences exist between responding for a CRf previously paired with a natural, primary reinforcer (water) using Pavlovian conditioning and seeking responses made during reinstatement tests following self-administered reward.

The fact that H-IA or H-IC animals do not respond more for the CRf is puzzling, as high impulsivity is considered a risk factor for developing addiction and CSs invigorate craving and drug-seeking in addicts (Tiffany and Carter, 1998; Volkow et al, 2006). A simple explanation could be related to the type of reinforcer used in the present study (water). The relationship between high impulsivity and CSs associated with natural reinforcers may differ from drugreinforcers. A more speculative explanation could be related to the CS. Similar to the cues used during self-administration studies in animals, the specific CSs which induce craving or drug-seeking in human subjects may be important in maintaining this relationship. If interaction with drugpaired stimuli directly lead to drug-intake in the past (eg, a lit cigarette, a pipe, and so on), then highly impulsive subjects may be more susceptible to those cues. However, similar to the CS in the present study, if the stimuli are only just associated with the drug reward (eg, the type of music playing during drug-taking), then high impulsivity may be less important. Future studies should be conducted to determine if the type of stimulus alters the relationship between impulsivity and drug-seeking and if addicts also have altered responses to non-drug-paired CSs.

Responding for a CRf can be used as a measurement of the amount of salience attributed to the CS. Using a different paradigm, interactions with a CS are recorded as a measurement of incentive salience (Robinson and Flagel, 2009). Similar to results presented here, levels of impulsive choice were similar between animals that primarily interacted with the CS (ie, sign-trackers) or those that primarily interacted with the reward receptacle (ie, goal-trackers; Lovic et al, 2011). In contrast, sign-trackers were more impulsive on two measures of impulsive action: premature responses on a simplified 5CSRTT (a 2CSRTT) and responding maintained by a differential reinforcement of low rates of responding (Lovic et al, 2011). However, a high degree of variance was noted; some sign-trackers were not very impulsive, whereas some goal-trackers were quite impulsive (Lovic et al, 2011). In addition, the simplified 2CSRTT may not be as sensitive to attentional and impulsive processes considering the reduced number of potential options for the stimulus. Furthermore, the ability to interact with the CS (illuminated lever) in studies measuring sign- and goaltracking may influence the amount of salience attributed towards the CS (Meyer et al, 2014). In the present study, interactions with the CS are less likely owing to the nature of the CS (light-tone). Nonetheless, as interactions with the CS were not recorded in the present study, it is unknown if H-IA rats in the present study were also sign-trackers.

The effect of amphetamine on delay-to-gratification was measured using a DDT (Experiment 2). Systemic administration of amphetamine increased impulsive choice (decreased choice of the large, delayed reward), similar to previous work (Cardinal et al, 2000; Evenden and Ryan, 1996). However, decreased impulsive choice has also been observed following a systemic injection of amphetamine (eg, Floresco et al, 2008; van Gaalen et al, 2006; Wade et al, 2000; Winstanley et al, 2003). The opposing effects of amphetamine on delay-discounting may depend on whether the delay was signaled with a cue. If a cue was present during the delay, amphetamine decreased impulsive choice, whereas if the delay was unsignalled (similar to the present study), amphetamine increased impulsive choice (Cardinal et al, 2000).

In Experiment 1, the duration of time animals were required to refrain from responding on the 5CSRTT was increased by lengthening the ITI. Similar to previous research (Dalley et al, 2007; Economidou et al, 2009), this manipulation increased impulsive action in all groups and $\mathrm{H}$-IA rats maintained the greatest number of premature responses. Administration of amphetamine also increased impulsive action in all groups. Amphetamine increases the release of DA as well as norepinephrine and serotonin. However, blockade of the norepinephrine (Fernando et al, 2012) or serotonin (Baarendse and Vanderschuren, 2012) transporters reduced impulsive action on the 5CSRTT. In contrast, administration of a selective DA transporter blocker increased premature responding in low and high impulsive subjects (Fernando et al, 2012). In addition, amphetamine's ability to enhance premature responding on the 5CSRTT is largely attributed to DA release in the NAc (Pattij and Vanderschuren, 2008; Pattij et al, 2007; Robbins, 2002). 
High impulsivity is an important risk factor for the development of addiction and increased relapse (Jupp and Dalley, 2014; Stevens et al, 2014). Notably, human subjects with a very high familial risk for developing an addiction exhibited decreased DA release in the ventral striatum compared with control subjects following ingestion of a low dose of amphetamine (Casey et al, 2014). In the present study, compared with L-IA rats, H-IA animals also demonstrated less NAc DA release following relatively low doses of amphetamine. Even though there was no apparent relationship between impulsive choice and responding for a CRf, compared with L-IC rats, H-IC rats also demonstrated decreased stimulated DA release $(0.6 \mathrm{mg} / \mathrm{kg}$ dose only). However, a possible caveat is that the order of amphetamine injections was not counterbalanced during the microdialysis testing. Therefore, an increased effect following the $0.6 \mathrm{mg} /$ $\mathrm{kg}$ dose should be interpreted with caution. Nonetheless, blunted DA release in the ventral striatum-specifically the NAc-may not only indicate a lack of impulse control but could also suggest an increased risk of developing an addiction.

In summary, low impulsive action, but not impulsive choice, appears to predict enhanced responding for CRf, indicative of greater attribution of motivational salience toward the CS. This finding is supported by an initially greater rate of responding for the CRf in L-IA rats compared with I-IA and H-IA groups and augmented effects of amphetamine to enhance responding for a CRf. Consistent with our behavioral findings, amphetamine resulted in greater DA release in the NAc in L-IA rats compared with H-IA rats. Therefore, heightened impulsivity does not result in an abnormal attribution of salience to all CSs and reduced DA release in the NAc may be an important biomarker of heightened impulsivity.

\section{FUNDING AND DISCLOSURE}

The authors declare no conflict of interest.

\section{ACKNOWLEDGMENTS}

We thank Dr Doug Funk for providing expert technical assistance in maintaining the HPLC equipment. This work was funded by an operating grant from the Canadian Institutes of Health Research (CIHR) to PJF. FDZ is supported by a CIHR Postdoctoral Fellowship. FDZ previously consulted for Intervivo Solutions, on an unrelated matter. Portions of this work were presented as posters at the 2013 Society for Neuroscience meeting and the 2014 International Behavioral Neuroscience Society meeting.

\section{REFERENCES}

Baarendse PJJ, Vanderschuren LJMJ (2012). Dissociable effects of monoamine reuptake inhibitors on distinct forms of impulsive behavior in rats. Psychopharmacology 219: 313-326.

Browne JD, Soko AD, Fletcher PJ (2014). Responding for conditioned reinforcement in C57BL/6 and CD-1 mice, and Sprague-Dawley rats: effects of methylphenidate and amphetamine. Psychopharmacology 231: 4503-4516.
Buckholtz JW, Treadway MT, Cowan RL, Woodward ND, Li R, Ansari MS et al (2010). Dopaminergic network differences in human impulsivity. Science 329: 532.

Caggiula AR, Donny EC, White AR, Chaudhri N, Booth S, Gharib MA et al (2001). Cue dependency of nicotine self-administration and smoking. Pharmacol Biochem Behav 70: 515-530.

Cardinal RN, Robbins TW, Everitt BJ (2000). The effects of d-amphetamine, chlordiazepoxide, alpha-flupenthixol and behavioural manipulations on choice of signalled and unsignalled delayed reinforcement in rats. Psychopharmacology 152: 362-375.

Casey KF, Benkelfat C, Cherkasova MV, Baker GB, Dagher A, Leyton M (2014). Reduced dopamine response to amphetamine in subjects at ultra-high risk for addiction. Biol Psychiatry 76: 23-30.

Comings DE, Blum K (2000). Reward deficiency syndrome: genetic aspects of behavioral disorders. Prog Brain Res 126: 325-341.

Dalley JW, Fryer TD, Brichard L, Robinson ESJ, Theobald DEH, Lääne $\mathrm{K}$ et al (2007). Nucleus accumbens D2/3 receptors predict trait impulsivity and cocaine reinforcement. Science 315 : 1267-1270.

Di Ciano P, Everitt BJ (2004). Conditioned reinforcing properties of stimuli paired with self-administered cocaine, heroin or sucrose: implications for the persistence of addictive behaviour. Neuropharmacology 47(Suppl 1): 202-213.

Diergaarde L, Pattij T, Nawijn L, Schoffelmeer ANM, De Vries TJ (2009). Trait impulsivity predicts escalation of sucrose seeking and hypersensitivity to sucrose-associated stimuli. Behav Neurosci 123: 794-803.

Diergaarde L, Pattij T, Poortvliet I, Hogenboom F, de Vries W, Schoffelmeer ANM et al (2008). Impulsive choice and impulsive action predict vulnerability to distinct stages of nicotine seeking in rats. Biol Psychiatry 63: 301-308.

Downs B, Oscar-Berman M, Waite R, Madigan M, Giordano J, Beley $\mathrm{T}$ et al (2013). Have we hatched the addiction egg: reward deficiency syndrome solution system ${ }^{\mathrm{Tm}}$. J Genet Syndr Gene Ther 4: 14318.

Economidou D, Pelloux Y, Robbins TW, Dalley JW, Everitt BJ (2009). High impulsivity predicts relapse to cocaine-seeking after punishment-induced abstinence. Biol Psychiatry 65: 851-856.

Evenden JL, Ryan CN (1996). The pharmacology of impulsive behaviour in rats: the effects of drugs on response choice with varying delays of reinforcement. Psychopharmacology 128: 161-170.

Feenstra M. Chapter 4.2 Microdialysis of dopamine and norepinephrine during conditioning and operant behavior. In: Westerink BHC, Cremers TIFH (eds). Handbook of Microdialysis Methods, Applications and Perspectives, Elsevier, Amsterdam: Oxford, 2007, pp 317-350.

Fernando ABP, Economidou D, Theobald DE, Zou M-F, Newman AH, Spoelder $M$ et al (2012). Modulation of high impulsivity and attentional performance in rats by selective direct and indirect dopaminergic and noradrenergic receptor agonists. Psychopharmacology 219: 341-352.

Floresco SB, Tse MT, Ghods-Sharifi S (2008). Dopaminergic and glutamatergic regulation of effort- and delay-based decision making. Neuropsychopharmacology 33: 1966-1979.

Joutsa J, Voon V, Johansson J, Niemelä S, Bergman J, Kaasinen V (2015). Dopaminergic function and intertemporal choice. Transl Psychiatry 5: e491.

Jupp B, Dalley JW (2014). Convergent pharmacological mechanisms in impulsivity and addiction: insights from rodent models. Br J Pharmacol 171: 4729-4766.

Justice JB Jr (1993). Quantitative microdialysis of neurotransmitters. J Neurosci Methods 48: 263-276.

Lovic V, Saunders BT, Yager LM, Robinson TE (2011). Rats prone to attribute incentive salience to reward cues are also prone to impulsive action. Behav Brain Res 223: 255-261. 
McDonald J. Handbook of Biological Statistics. 3rd edn. Sparky House Publishing: Baltimore, MD, USA, 2014.

McNamara R, Dalley JW, Robbins TW, Everitt BJ, Belin D (2010). Trait-like impulsivity does not predict escalation of heroin self-administration in the rat. Psychopharmacology 212: 453-464.

Meyer PJ, Cogan ES, Robinson TE (2014). The form of a conditioned stimulus can influence the degree to which it acquires incentive motivational properties. PLoS One 9: e98163.

Olmstead MC, Robbins TW, Everitt BJ (1998). Basal forebrain cholinergic lesions enhance conditioned approach responses to stimuli predictive of food. Behav Neurosci 112: 611-629.

Pattij T, Janssen MCW, Vanderschuren LJMJ, Schoffelmeer ANM, van Gaalen MM (2007). Involvement of dopamine D1 and D2 receptors in the nucleus accumbens core and shell in inhibitory response control. Psychopharmacology 191: 587-598.

Pattij T, Vanderschuren LJMJ (2008). The neuropharmacology of impulsive behaviour. Trends Pharmacol Sci 29: 192-199.

Perry JL, Larson EB, German JP, Madden GJ, Carroll ME (2005). Impulsivity (delay discounting) as a predictor of acquisition of IV cocaine self-administration in female rats. Psychopharmacology 178: 193-201.

Robbins TW (2002). The 5-choice serial reaction time task: behavioural pharmacology and functional neurochemistry. Psychopharmacology 163: 362-380.

Robbins TW, Watson BA, Gaskin M, Ennis C (1983). Contrasting interactions of pipradrol, d-amphetamine, cocaine, cocaine analogues, apomorphine and other drugs with conditioned reinforcement. Psychopharmacology 80: 113-119.

Robinson TE, Flagel SB (2009). Dissociating the predictive and incentive motivational properties of reward-related cues through the study of individual differences. Biol Psychiatry 65: 869-873.

Rose JE, Behm FM, Westman EC, Johnson M (2000). Dissociating nicotine and nonnicotine components of cigarette smoking. Pharmacol Biochem Behav 67: 71-81.
Stevens L, Verdejo-Garcia A, Goudriaan AE, Roeyers H, Dom G, Vanderplasschen W (2014). Impulsivity as a vulnerability factor for poor addiction treatment outcomes: a review of neurocognitive findings among individuals with substance use disorders. J Subst Abuse Treat 47: 58-72.

Taylor JR, Robbins TW (1986). 6-Hydroxydopamine lesions of the nucleus accumbens, but not of the caudate nucleus, attenuate enhanced responding with reward-related stimuli produced by intra-accumbens d-amphetamine. Psychopharmacology 90: 390-397.

Tiffany ST, Carter BL (1998). Is craving the source of compulsive drug use? J Psychopharmacol 12: 23-30.

van Gaalen MM, van Koten R, Schoffelmeer AN, Vanderschuren LJ (2006). Critical involvement of dopaminergic neurotransmission in impulsive decision making. Biol Psychiatry 60: 66-73.

Volkow ND, Wang G-J, Telang F, Fowler JS, Logan J, Childress A-R et al (2006). Cocaine cues and dopamine in dorsal striatum: mechanism of craving in cocaine addiction. J Neurosci 26: 6583-6588.

Wade TR, de Wit H, Richards JB (2000). Effects of dopaminergic drugs on delayed reward as a measure of impulsive behavior in rats. Psychopharmacology 150: 90-101.

Winstanley CA, Dalley JW, Theobald DE, Robbins TW (2003). Global 5-HT depletion attenuates the ability of amphetamine to decrease impulsive choice on a delay-discounting task in rats. Psychopharmacology 170: 320-331.

Winterbauer NE, Balleine BW (2007). The influence of amphetamine on sensory and conditioned reinforcement: evidence for the re-selection hypothesis of dopamine function. Front Integr Neurosci 1: 9.

Zeeb FD, Floresco SB, Winstanley CA (2010). Contributions of the orbitofrontal cortex to impulsive choice: interactions with basal levels of impulsivity, dopamine signalling, and rewardrelated cues. Psychopharmacology 211: 87-98.

Supplementary Information accompanies the paper on the Neuropsychopharmacology website (http://www.nature.com/npp) 